\title{
Evaluation of an individual sleep intervention programme in people undergoing peritoneal dialysis treatment
}

Pia Yngman Uhlin, Anders Fernström, Sussanne Börjeson and Ulla Edéll-Gustafsson

\section{Linköping University Post Print}

N.B.: When citing this work, cite the original article.

This is the authors' version of the following article:

Pia Yngman Uhlin, Anders Fernström, Sussanne Börjeson and Ulla Edéll-Gustafsson, Evaluation of an individual sleep intervention programme in people undergoing peritoneal dialysis treatment, 2012, Journal of Clinical Nursing, (21), 23-24, 3402-3417.

which has been published in final form at:

http://dx.doi.org/10.1111/j.1365-2702.2012.04282.x

Copyright: Blackwell Publishing http://www.blackwellpublishing.com/

Postprint available at: Linköping University Electronic Press http://urn.kb.se/resolve?urn=urn:nbn:se:liu:diva-68040 
Title: Evaluation of an individual sleep intervention programme in people undergoing peritoneal dialysis treatment

Authors: Yngman-Uhlin Pia ${ }^{1,2}$, Fernström Anders ${ }^{2}$, Börjeson Sussanne ${ }^{1}$, Edéll-Gustafsson Ulla $^{1}$

${ }^{1}$ Department of Medical and Health Sciences, Division of Nursing Science, Faculty of Health Sciences, Linköping University, Linköping, Sweden

${ }^{2}$ Department of Nephrology, County council of Östergötland, Linköping, Sweden

Acknowledgement: We thank the PD-nurses: Berith Boo, Karin Berggren, Birgitta Lundberg, Annelie Ljungdal-Jonsson, Anette Strömberg and physician Mattias Alvunger at the participating clinics for assistance with patient recruitment. Tanks, also to physician Martin Ulander, Department of Neurophysiology at Linköping University Hospital, for support in sleep medicine matters.

\section{Contributions}

Study design: PY-U, UE-D, AF. Data collections and analysis: PY-U. Manuscript preparation: PY-U, AF, SB, UE-G.

Founding of support: Research Council of South-East of Sweden (FORSS) No; 37121, 87301

Disclosures: We hereby declare that no financial conflict of interest exists.

\section{Corresponding author:}

Pia Yngman-Uhlin, RN, MSc, PhD-student Linköping University

Department of Medical and Health Sciences

Division of Nursing Sciences

SE-581 85 Linköping, SWEDEN e-mail: pia.yngman.uhlin@liu.se telephone: +46101037792

fax: +4613227692

Running title: Sleep hygiene in people on peritoneal dialysis 


\section{ABSTRACT}

Aims and objectives: This study aimed to evaluate effects of a non-pharmacological intervention on sleep, activity and fatigue in patients receiving peritoneal dialysis by the use of both actigraphy registration and self-assessed questionnaires.

Background: Insomnia is estimated to affect up to $60 \%$ of hemo- and peritoneal dialysis patients. It is associated with two common uremic symptoms, pruritus and restless legs syndrome. To our knowledge no interventions have been evaluated by actigraphy.

Design: A prospective multiple baseline single-case experimental design.

Methods: Two women and seven men with sleep problems, 48-77 years, treated with PD participated in a 17-week study from January 2009 to February 2011. Two interventions were separately implemented. First, a pressure relieving mattress and second, a four week individual sleep hygiene and sleep scheduling intervention. The two interventions were evaluated both objectively by actigraphy and subjectively by questionnaires.

Results: A total of 315 sleep-wake cycles from nine individuals were evaluated. Three patients improved clinically significantly in five or more of the nine outcomes sleep onset latency, nocturnal sleep duration, numbers and duration of napping, movement and fragmentation index, number of steps, metabolic equivalent unit, sleep efficiency and fatigue. The other six patients also showed improvements but to a lesser degree. Physical activity advice was the intervention that yielded most sleep improvements.

Conclusions: This study illuminates the need for regular assessment of sleep and tiredness. It also demonstrates how a non-pharmacological treatment and self-management can be applied whit renal supportive care to improve sleep quality.

Relevance to clinical practice: This study is a clinical example of a non-pharmacological intervention with supportive care and self-management. This model can improve health and 
reduce the pharmacological burden since hypnotics can be replaced by sleep hygiene self-care activities.

Keywords: Peritoneal dialysis, renal supportive care, sleep hygiene, non-pharmacological treatment, insomnia, fatigue, self-care

\section{INTRODUCTION}

Sleep problems are estimated to affect up to $60 \%$ of people undergoing hemo- and peritoneal dialysis (PD) treatment (Al-Jahdali et al. 2010, Novak et al. 2006, Yngman-Uhlin \& EdellGustafsson 2006). Insomnia is one of the dominating sleep problems varying from persistent trouble falling asleep, difficulties in maintaining sleep and too early morning awakening combined with daytime symptoms (WHO 1993). Insomnia is also associated with two prevalent and often persistent uremic symptoms in people with PD; restless legs syndrome (RLS) 21.5-47\% (Araujo et al. 2010, Pisoni et al. 2006, Sabry et al. 2010) and pruritus 3942\% (Pisoni et al. 2006, Yngman-Uhlin \& Edell-Gustafsson 2006). Furthermore a previous study by our research group revealed that difficulties finding a good sleeping position explained sleep quality in 56\% of the patients (Yngman-Uhlin \& Edell-Gustafsson 2006). People receiving PD treatment at home require self-care management abilities. A complex series of behaviours take place in the self-care management (Riegel \& Weaver 2009) which ean be altered by decreased sleep quality. The complex series of behaviours which take place in self-care management (Riegel \& Weaver 2009) may be adversely affected by a decreased sleep quality. Patients need to evaluate their fluid balance, perform treatment in an aseptic manner and be ready to act if complications occur. Decreased sleep quality results in sleepiness, tiredness as well as impairments of attention and concentration abilities (Drummond et al. 2004) which can reduce self-care skills. Further, decreased sleep quality 
has been reported to have a negative effect on health-related quality of life (HRQoL) in patients with PD (Bilgic et al. 2008, Yang et al. 2007).

\section{Background}

Many people on dialysis treatment are physically deconditioned from the chronicity of their disease and co-morbidities (Davisen \& Jhangri 2010, Murtagh et al. 2007), making them vulnerable to sleep problems, as dialysis alters both metabolic (Knutson 2007) and immunological (Chim et al. 2009, Redwine et al. 2000) functions. This is serious since the two most common causes of death in people with dialysis are cardiovascular diseases and infections (Kendrick \& Teitelbaum 2010).-Many people on dialysis treatment are physically deconditioned from the chronicity of their disease and co-morbidities (Davison \& Jhangri 2010, Murtagh et al. 2007), making them vulnerable to sleep problems. Loss of deep sleep i.e. fragmented sleep, alters both metabolic (Knutson 2007) and immunological (Chiu et al. 2009, Redwine et al. 2000) functions. This is serious since cardiovascular diseases and infections are the two most common causes of death in patients undergoing dialysis (Kendrick \& Teitelbaum 2010).

In general hypnotics is the most frequently used treatment for insomniacs in general (Lieberman 2007, Neutel \& Patten 2009) but there are side-effects such as daytime sleepiness, dependency and drug-drug interactions (Barbera \& Shapiro 2005). Non-pharmacological treatment such as sleep hygiene interventions (Morin et al. 1999), daytime sleep restrictions and bright-light therapy (Fetveit \& Bjorvatn 2004) has been evaluated in a nursing home population with successful improvements of sleep onset. This might be the treatment of choice for people with self-care management abilities and with alterations in drug absorption, drug metabolism and, in many cases, polypharmacy. Renal supportive care can be used for 
complex self-care management (Noble et al. 2007) in PD patients with sleep problems. Sleep quality has shown to be improved by increased activity in people undergoing haemodialysis (Tentori et al. 2010) and long term effects of sleep behavioural therapy has shown to be more effective than medication in older people (Morin et al. 1999).

There is a knowledge gap concerning sleep problems among PD patients and sleep intervention studies have been requested. To our knowledge only one research group has performed a non-pharmacological intervention study, i.e. cognitive behavioural therapy in combination with a sleep hygiene intervention, with a positive effect on insomnia in PD patients (Chen et al. 2008). This said, the intervention effects on sleep parameters were solely assessed by subjective measurement i.e. questionnaires. Further, there is only one study that has used one-week actigraphy registration in a small group of PD patients ( $\mathrm{n}=6)$ (Koch et al. 2010). This is the best way of evaluating the sleep-wake cycle for an extended period of time in the home environment. In addition physical activity can concurrently be registered.

\begin{abstract}
Aim
The aim of this study was to evaluate the effects of a nurse-led non-pharmacological intervention on sleep, activity and symptoms of fatigue in PD patients by the use of both actigraphy registration and self-assessed questionnaires.
\end{abstract}

\title{
THE STUDY
}

\section{Design}

This study had a prospective multiple baseline single-case, AABBC experimental design with five repeated measure phases in which each participant served as his/her own control (table 1). The A phases were two multiple baseline data collections, the B phases were intervention 
phases and the $\mathrm{C}$ phase was a non-treatment phase with a follow-up. This design was chosen because of the patient-centered focus and the near-clinical situation. It is suitable for research in behavioural interventions that directly measure therapeutic and individual outcomes (Kazdin 1982). it was used since a behavioural treatment intervention effect i.e. education, is irreversible (Morgan \& Morgan 2009).

-Insert table 1 here-

\section{Participants}

Twenty eight eligible patients from two university hospitals and two general hospitals in the southeast of Sweden were invited to participate in the study from January 2009 to September 2010. Data collection ended in February 2011. The patients were invited one by one due to the logistical availability of the study equipment. Inclusion criteria were: over 18 years of age, self-managed peritoneal dialysis treatment at home for more than two months, medically stable at the time of inclusion, listed for transplantation $<6$ months and having indications of sleep problems with daytime symptoms. Nineteen patients accepted participation, although four did not actually have persistent sleep problems and were not included. During the baseline phase two patients dropped out due to disease-related complications, two underwent transplantation and two died. Finally, two women and seven men (Table 2) fulfilled the 17week study plan. Patients who were on hypnotics treatment before study inclusion maintained the same dose during the study.

-Insert table 2 here-

\section{Interventions}

The interventions were performed in two phases. First, a mattress (Tempur®) made of polyurethane foam was offered to all participants for two consecutive weeks. This intervention aimed to evaluate if the difficulties finding a good sleeping position could be reduced, a previously detected problem by our research group (Yngman-Uhlin \& Edell- 
Gustafsson 2006). Second, an individual sleep hygiene advice program, which was designed after careful review of the collected baseline data, was put into practice. Sleep hygiene advice, sleep scheduling and bright light therapy were recommended depending on the type of sleep problem (Morin \& Espie 2004) (Table 3).

In sleep hygiene advice, sleep-related lifestyle and sleep context can together form a set of sleep-related behaviours that may be suitable for sleep (Morin \& Espie 2004), for instance reducing disturbing noise at bedtime or increase daytime activity. Activity could be any physical activity such as walking, gardening, and light exercise. Stretching or light exercise can be used to reduce RLS (Aukerman et al. 2006). Further, avoiding caffeine intake since this can delay sleep onset or cause RLS, was recommended. Sleep scheduling was recommended to establish regular sleep habits. This aimed to build up the sleep drive and increase sleep efficiency by spending less time in bed and increase sleep-wake specificity by avoiding sleep in the daytime (Morin \& Espie 2004). For misaligned sleep-wake phases, bright light therapy (Sunrise system 320® Bright LED portable light box, 10.000 Lux) was recommended in the morning for 30-45 minutes, applied at 30-50 centimeters distance to inhibit the internal secretion of melatonin (Gooley 2008). Numbers of sleep promoting recommendations were limited to no more than three for each patient.

-Insert table 3 here-

\section{Data collection}

The interventions were evaluated by nine outcome variables; 1) sleep onset latency, 2) nocturnal sleep duration, 3) numbers of and 4) duration of napping, 5) movement and fragmentation index (MFI), 6) number of steps, 7) Metabolic Equivalent Unit (METs), 8) sleep efficiency and 9) fatigue. Data were collected both for assessment of sleep problems at baseline and for evaluation of the interventions (Table 1). This was done objectively by 
actigraphy registration and subjectively by four questionnaires and a sleep diary. Medical data were collected from the patients' records.

The objective assessment consisted of a wireless wearable body monitor, SenseWear® pro actigraph (Bodymedia, Pittsburgh, PA). The device is designed to be worn comfortably at the upper arm under clothing in a normal everyday environment. One-week registrations were recorded and ended each of the five study phases of four weeks (Table 1). Four sensors registered physiological body signals every minute: skin temperature, galvanic skin response, heat flux and a 2-axis accelerometer. Data were thereafter processed by the software program SensWear ${ }^{\circledR} 6.1$ (Bodymedia Inc.) where the sensors' information combined with the algorithms calculated and reported: METs (Kcal/Kg/hour), total number of steps, sleep duration, time lying down and sleep efficiency index (\%), i.e. the amount of time in bed actually spent sleeping. A sleep efficiency index above $85 \%$ is generally considered to be good. For each night a movement index (MI) and fragmentation index (FI) were calculated. MI was obtained by dividing every minute of movement with total time in bed multiplied by 100. FI was the ratio of one-minute immobility to the total number of immobility phases multiplied by 100. By adding MI and FI, a global index for disrupted sleep was obtained; movement and fragmentation index (MFI) (Aubert-Tulkens et al. 1987). An index of more than 50 represents serious fragmentation whereas below 20 represents well consolidated sleep (Camtech 2007). In addition, visual graphs of the sleep wake-cycles were provided by the program. These were used to motivate and educate the patients when providing them with sleep hygiene advice. Validations between actigraphy and polysomnography have shown high sensitivity (89\%) and specificity (95\%) for diagnosing sleep apnoea (Aubert-Tulkens et al. 1987, Stepanski 2002) as well as accuracy in number of nocturnal awakenings, awakenings after sleep onset, total sleep time and sleep efficiency in insomnia patients (Lichstein et al. 2006) 
The Functional Assessment of Chronic Illness Therapy (FACIT)- fatigue scale was used to measure the degree of fatigue (Yellen et al. 1997). The 13 items were answered in a five-point scale from "not at all (0) to "very much" (4). The scale ranges from zero to 52, where a lower score indicates a higher degree of fatigue. The cut-off score is set to 43 in a general population (Yellen et al. 1997). Examples of the questions are: "I feel too weak" and "I am able to do my usual activities". The items in the scale essentially assess activity i.e. functional fatigue. The scale has been tested for internal consistency where Cronbach's alpha was 0.94 (Yellen et al. 1997).

To evaluate sleep problems at baseline, a study-specific sleep diary was completed during the five weeks of actigraphy registration. This was used as a complement for interpreting the actigraphy data during the study. Habitual sleep information referring to the last four weeks was assessed by a modified version of the Uppsala Sleep Inventory (USI) (Hetta et al. 1985) (Yngman-Uhlin \& Edell-Gustafsson 2006). The health profile was assessed by SF-36 (Sullivan et al. 1995, Ware \& Sherbourne 1992), which is the most frequently used questionnaire for evaluation of health outcome (Garratt et al. 2002). It assesses eight health domains where a higher score indicates better health (0-100). The severity of restless legs was assessed by a validated scale; The International Restless Legs Scale (IRLS) which has previously shown a good internal consistency (Cronbach's alpha 0.76-0.81) (Abetz et al. 2006, Walters et al. 2003).

\section{Procedure}

Eligible patients matching the inclusion criteria were identified by the PD nurses at the participating clinics. and They were then sent an invitation letter which also contained a few 
questions about sleep quality and daytime symptoms. If the patients experienced persistent sleep problems and agreed to participate they were scheduled for a 17-weeks registration-, and intervention program. The study is divided into five phases and each phase ends with an actigraphy registration (Table 1). During an initial home visit, made by the first author, a sleep history was obtained by a study-specific standardised outline plan for sleep history assessment inspired by Morin and Espie (Morin \& Espie 2004). At this first home visit the actigraph and the questionnaires were delivered by the first author but were thereafter sent by post. At the second home visit, in study phase three, the mattress which was the first intervention was delivered. The individual sleep hygiene intervention plans, based on baseline data, were discussed with a neurophysiologist before being presented and implemented in concordance with the patients at the third home visit. During the second intervention phase the first author had a supporting and coaching function by telephone twice weekly. A final follow-up registration was performed four weeks after the completion of the sleep hygiene intervention.

\section{Ethical considerations}

The study was performed in accordance with the Declaration of Helsinki (WMA 2008) after approval by the Regional Ethical Review Board at the faculty of Health Sciences, Linköping University. Written informed consent were obtained from all participants.

\section{Data analysis}

Data are presented in accordance with single-case studies (Morgan \& Morgan 2009) in tables and as graphs with each patient represented over a timeline. Changes of the outcome variables of half a standard deviation $(1 / 2 \mathrm{SD})$ or more compared to the mean value of the two baselines, were applied as an indicator for an individual clinically significant change (Frost et 
al. 2007). This was used to avoid reflections of normal fluctuations. To evaluate the effect of the intervention at group level non-parametric statistics, Friedman's test followed by Wilcoxon signed rank test were used (Field 2009). Bonferroni was performed as a post hoc test where a significant level was set to 0.016 , otherwise 0.05 was accepted. Microsoft Office Excel 2007 and PASW statistical software, version 18.0 were used for graphs and statistical analyses.

\section{RESULTS}

Three of nine patients ( $B, G$ and $I)$ improved clinically significantly in five or more of the nine outcome variables when comparing baseline and the final week of the second intervention i.e. individual sleep hygiene advice (table 4). For three of the patients unexpected events not related to the study occurred during the intervention period (Table 3). Two of the participants $(F, H)$ did not want to take part in the first intervention i.e. sleep on the mattress and two took the mattress away after only two nights $(I, G)$ due to discomfort.

-Insert table 4 here-

\section{Sleep duration}

All patients except patient $D$ had a sleep duration shorter than 6 hours (Figure 1) at baseline. Patients $G, C$ and $I$ increased their sleep duration-elinically significantly during the second intervention compared to baseline (Figure 1), but none of them improved their sleep duration to 6 hours or more during the intervention. Patient $D$ did decrease nocturnal sleep duration but this was also an improvement since the sleep duration at baseline two was high, 13 hours and 6 minutes.

-Insert figure 1 and 2 here-

Three patients, patients F,G and I, elinieally significantly reduced their napping time during the second intervention. Patient $G$ and $I$ reduced their daytime sleep to less than 30 minutes. 
In total, six patients slept less than 30 minutes during the day at the follow-up phase $D-I$ (Figure 1).

\section{Activity}

Seven patients were advised to increase physical activity and of these, $B, G$ and $I$ improved the number of steps elinically significantly compared to baseline. METs were elinieally significantly increased for the same patients (Figure 2).

-Insert figure 3 here-

\section{Sleep efficiency and sleep fragmentation}

Sleep efficiency was significantly elinically improved for patients $A, G, H$ and $I$ compared to baseline, only two of the patients were above the recommended $85 \% ; B$ and $D$ (Fig 3). Three patients $A, D$ and $I$ improved elinically significantly in MFI and finished below index 20 .

\section{Fatigue}

All patients except one $(H)$ were classified as having fatigue at baseline. After the sleep hygiene intervention, two patients were above the cut off score 43. Clinically Significant improvement in four of the patients $B, D, E$ and $H$ was shown between baseline and study phase 4 (Figure 3).

Health profiles were assessed at baseline 1 and at follow-up. Of the three patients $B, G$ and $I$ who improved in five or more of the outcome variables, only $G$ had visual improvements in one of eight dimensions in SF-36, vitality, but decreased in social function, role emotional and mental health (Figure 4). 
Of the five patients who fulfilled the mattress intervention, improvements were found in three of the variables for patients $A$ and $D$. Patient $C$ and $E$ improved in one variable (Table 4). At the follow-up, patient $I$ had maintained the improvements in five of the outcome variables four weeks after the intervention period (Table 4).

Analysis at group level showed no statistical significances in any variables between any of the study phases. The sleep efficiency index improved in study phase 4 compared to 3 ( $p=0.028$ ), but this was not statistically significant after correction with Bonferroni.

\section{DISCUSSION}

The present study demonstrates that individual, nurse-led, non-pharmacological, sleep interventions improved sleep and daytime activities in patients on PD treatment in a nearclinical situation. By supporting the patients to make simple, inexpensive lifestyle changes three out of nine, patients $B, G$ and $I$, stopped or reversed the progression of a negative sleep behaviour and improved in five or more of the nine outcome variables. In addition, the other six patients showed improvements which are notable in this multi-disease and frail patient group. Although the patients did not reach the recommended sleep duration of six hours they may have obtained benefits from their improved sleep.

This study also shows the importance of systematic assessment in daily clinical practice. Based on this data, sleep advice i.e. behavioural interventions can be provided, thus possibly resulting in an improved daily life for these frail patients. Behavioural treatment has been described as being sustainable over time in people with insomnia in late-life, compared to hypnotics which was more effective in the short term (Morin et al. 1999). PD nurses are a group of healthcare providers with a unique position for supporting self-care management. A 
carefully conducted sleep history can provide important information that is frequently overlooked in the standard anamnesis. This is especially important in patients on PD since they have a high prevalence of sleep problems (Al-Jahdali et al. 2010, Novak et al. 2006, Yngman-Uhlin \& Edell-Gustafsson 2006). For patients with indications of sleep problems we suggest that an individual sleep assessment followed up with targeted interventions should be prescribed as a regular part of their medical care.

All patients in the study had disturbed sleep and fulfilled the criteria for insomnia (WHO 1993). At baseline, eight of the nine patients had shorter nocturnal sleep duration than the recommended six hours (WHO 1993). Two of the patients decreased their napping time significantly; both of them had naps of less than 30 minutes in study phases 4 and 5. For insomniacs, daytime restrictions often limit naps to no more than 30 minutes and not later than $3.00 \mathrm{pm}$ (Morin et al. 1999). From our results we identified one patient $(G)$ who succeeded significantly both in reducing napping time and increasing nocturnal sleep duration. Although reduced napping time is mostly beneficial for nocturnal sleep, one study has suggested that napping time contributes to significant increase of the 24-hour quota (Campbell et al. 2005). This must be considered when deciding if restrictions should be prescribed in PD patients with such decreased nocturnal sleep duration.

Sleep efficiency was improved for as many as five of nine patients. Sleep efficiency is an important variable measuring the amount of time in bed actually spent sleeping. Three patients improved in MFI and reached values below 20. This is important since fragmented sleep is a serious problem. It reduces restorative deep sleep i.e. sleep quality with metabolic (Knutson 2007) and immunologic (Chiu et al. 2009, Redwine et al. 2000) alterations. 
All three patients who succeeded in improving five or more of the nine outcome variables were prescribed increased physical activity, i.e. number of steps and METs, which was also the most successful intervention in the whole group. Regarding the activity, two of the nine patients had a stationary exercise bike at home. The actigraph applied at the upper arm does not measure such activity and therefore energy expenditure was an important additional outcome variable. Nevertheless, those who had clinically significantly increased METs also had an increased number of steps.

During the last intervention week self-reported fatigue was low in all patients except $F$ and $H$. We have previously reported a high prevalence of fatigue $88 \%$ in the PD population from the same geographic area as the patients in this study. (Yngman-Uhlin \& Edell-Gustafsson 2006), This is also supported by another study (Ossareh 2003). Fatigue was improved in four patients, but only one who improved in five or more variables also improved in fatigue. One explanation could be that the experience of decreased fatigue could be delayed or in need of an extended intervention period. Fatigue could also be the result of other disease-related factors than sleep disturbances.

The three patients with the lowest numbers of improved outcome variables were patients $D, F$ and $H$. They were the youngest and they also scored the highest on the SF-36 (widest areas). They did not have the most deteriorated sleep at baseline and might have had other expectations of their sleep. This may suggest that they needed another kind of intervention or support.

It is difficult to evaluate the mattress interventions in this study since four of the patients had not used the mattress at the time of the first intervention assessment. For those who fulfilled 
the two-week mattress intervention few variables were changed. We assumed that the mattresses might facilitate a better anatomic position since they mould themselves to the exact shape of the body. Another study demonstrated fewer twists from side to side with a polyurethane mattress (Bergholdt et al. 2008) which might indicate less fragmented sleep. However, this was not detected in the present study.

The variables which started to improve during study phase 3 (mattress), even for patients who declined to use or removed the mattress, might be explained by other effects e.g. the Hawthorne effect, which is defined as the additional response from increased attention provided from the study situation (Wolfe \& Michaud 2010). Likewise, in clinical situations improvements have been explained by the nurse-dose effect which refers to the amount of nurse time and number of contacts (Brooten \& Youngblut 2006). The coaching function by the first author might have been an important intervention since telephone follow-ups have been demonstrated to improve adherence to self-care in heart failure patients (Riegel et al. 2002, Wong et al. 2010). Nevertheless this is analogous to a clinical situation and may be an important intervention in itself.

Two of the patients in this study suffered severe joint pain during the night. It can only be presumed that an optimized treatment for the pain in combination with sleep hygiene interventions might have resulted in a better sleep outcome. For more intensive interventions other symptom management strategies have to be considered in parallel. In the assumptions behind the theory of unpleasant symptoms, Lenz and co-workers state that multiple symptoms may be experienced to have a multiplicative effect and consequently, interventions of different kinds may be effective for more than one symptom (Lenz et al. 1997). This requires 
an awareness of symptoms related to poor sleep and sleep disturbing factors which makes the healthcare providers better prepared to intervene in these conditions.

\section{Study limitations}

Nine patients fulfilled the 17-week study plan. In traditional experimental studies this seems a small number of participants. However, in single-case studies, three or more subjects is considered a good number because the second, the third and so on are replications of the first subject which is a primary mechanism for establishing generality of empirical findings (Morgan \& Morgan 2009). We have objectively collected data from totally 315 sleep-wake cycles, i.e. 35 per patient, which is a strength in the study. Since there is no consensus on how to measure clinical significances, $1 / 2 \mathrm{SD}$ of change was used (Frost et al. 2007) and applied to define clinical significance in HRQoL measurements. However, estimations of that kind must be seen as a guidance to interpret the effect of the interventions rather than a rule (Sloan et al. 2005). In addition, some authors suggest that differences of less than $1 / 2$ SD may be considered as clinically significant (Cella et al. 2003).

Despite the fact that the inclusion criteria were strictly followed, the participants in this study were a very heterogeneous group with regard to lifestyle, habits, morbidity and life situation. Some of the participants were stressed due to social issues and progressing morbidity, which were not possible to control in the study. This may have affected the results, but not for all as patient $G$ improved in six of the nine outcome variables despite a serious family incident during the intervention. The individual intervention approaches also strengthen the study since they can be compared to a clinical situation. 
The interventions were limited to primarily sleep hygiene and sleep scheduling interventions which are two components in the concept of cognitive-behavioural therapy (CBT) for primary insomnia (Pinto et al. 2010). A broader approach i.e. a full CBT intervention might have been more beneficial. Adaptation to new routines, i.e. behavioural or lifestyles changes, might need more time than the 4-week program used. However, the patients in our study improved in a number of variables, but the effect could perhaps have been more profound and maintained for longer with an extended program.

This study demonstrates many clinically significant individual improvements despite no statistical significances on group level having appeared. Positive outcomes for separate individuals are not always visible in studies conducted at group level. Therefore, studies with different designs are meaningful. Further studies are needed to discover how Patients responsive to non-pharmacological interventions can be identified. Moreover, studies combining both non-pharmacological and pharmacological interventions are required in this population.

\section{CONCLUSION}

This study illuminates the need for regular assessment of sleep and tiredness and demonstrates how non-pharmacological treatment and self-management can be applied with renal supportive care to improve sleep quality.

\section{Conflict of interest}

The authors declare no conflict of interest.

\section{REFERENCES}


Abetz L, Arbuckle R, Allen RP, Garcia-Borreguero D, Hening W, Walters AS, Mavraki E \& Kirsch JM (2006): The reliability, validity and responsiveness of the International Restless Legs Syndrome Study Group rating scale and subscales in a clinical-trial setting. Sleep Med 7, 340-349.

Al-Jahdali HH, Khogeer HA, Al-Qadhi WA, Baharoon S, Tamim H, Al-Hejaili FF, AlGhamdi SM \& Al-Sayyari AA (2010): Insomnia in chronic renal patients on dialysis in Saudi Arabia. J Circadian Rhythms 8, 7.

Araujo SM, de Bruin VM, Nepomuceno LA, Maximo ML, Daher Ede F, Correia Ferrer DP \& de Bruin PF (2010): Restless legs syndrome in end-stage renal disease: Clinical characteristics and associated comorbidities. Sleep Med 11, 785-790.

Aubert-Tulkens G, Culee C, Harmant-Van Rijckevorsel K \& Rodenstein DO (1987): Ambulatory evaluation of sleep disturbance and therapeutic effects in sleep apnea syndrome by wrist activity monitoring. Am Rev Respir Dis 136, 851-856.

Aukerman MM, Aukerman D, Bayard M, Tudiver F, Thorp L \& Bailey B (2006): Exercise and restless legs syndrome: a randomized controlled trial. J Am Board Fam Med 19, 487-493.

Barbera J \& Shapiro C (2005): Benefit-risk assessment of zaleplon in the treatment of insomnia. Drug Saf 28, 301-318.

Bergholdt K, Fabricius RN \& Bendix T (2008): Better backs by better beds? Spine (Phila Pa 1976) 33, 703-708.

Bilgic A, Akman B, Sezer S, Ozisik L, Arat Z, Ozdemir FN \& Haberal M (2008): Predictors for quality of life in continuous ambulatory peritoneal dialysis patients. Nephrology (Carlton) 13, 587-592.

Brooten D \& Youngblut JM (2006): Nurse dose as a concept. J Nurs Scholarsh 38, 94-99.

Campbell SS, Murphy PJ \& Stauble TN (2005): Effects of a nap on nighttime sleep and waking function in older subjects. J Am Geriatr Soc 53, 48-53.

Camtech (2007): Actigraphy: Activity and Sleep Analysis. Cambridge Neurotechnology Ltd Information Bulletin No $4 B$.

Cella D, Zagari MJ, Vandoros C, Gagnon DD, Hurtz HJ \& Nortier JW (2003): Epoetin alfa treatment results in clinically significant improvements in quality of life in anemic cancer patients when referenced to the general population. J Clin Oncol 21, 366-373.

Chen HY, Chiang CK, Wang HH, Hung KY, Lee YJ, Peng YS, Wu KD \& Tsai TJ (2008): Cognitive-behavioral therapy for sleep disturbance in patients undergoing peritoneal dialysis: a pilot randomized controlled trial. Am J Kidney Dis 52, 314-323.

Chiu YL, Chuang YF, Fang KC, Liu SK, Chen HY, Yang JY, Pai MF, Peng YS, Wu KD \& Tsai TJ (2009): Higher systemic inflammation is associated with poorer sleep quality in stable haemodialysis patients. Nephrol Dial Transplant 24, 247-251.

Davison SN \& Jhangri GS (2010): Impact of pain and symptom burden on the health-related quality of life of hemodialysis patients. J Pain Symptom Manage 39, 477-485.

Drummond SP, Smith MT, Orff HJ, Chengazi V \& Perlis ML (2004): Functional imaging of the sleeping brain: review of findings and implications for the study of insomnia. Sleep Med Rev 8, 227-242.

Fetveit A \& Bjorvatn B (2004): The effects of bright-light therapy on actigraphical measured sleep last for several weeks post-treatment. A study in a nursing home population. $J$ Sleep Res 13, 153-158.

Field A (2009) Discovering statistics using SPSS (and sex and drugs and rock'n'roll), 3 edn. Oriental Press, Dubai, p. 816.

Frost MH, Bonomi AE, Cappelleri JC, Schunemann HJ, Moynihan TJ \& Aaronson NK (2007): Applying quality-of-life data formally and systematically into clinical practice. Mayo Clin Proc 82, 1214-1228. 
Garratt A, Schmidt L, Mackintosh A \& Fitzpatrick R (2002): Quality of life measurement: bibliographic study of patient assessed health outcome measures. BMJ 324, 1417.

Gooley JJ (2008): Treatment of circadian rhythm sleep disorders with light. Ann Acad Med Singapore 37, 669-676.

Hetta J, Almqvist M, Ågren H, Hambert G, Liljenberg G \& Roos BE (1985) Prevalence of sleep disturbances and related symptoms in a middle-aged Swedish population. Gustaf Fischer-Verlag, Stuttgart.

Kazdin AE (1982) Single-Case research designs. Oxford University Press, New York.

Kendrick J \& Teitelbaum I (2010): Strategies for improving long-term survival in peritoneal dialysis patients. Clin J Am Soc Nephrol 5, 1123-1131.

Knutson KL (2007): Impact of sleep and sleep loss on glucose homeostasis and appetite regulation. Sleep Med Clin 2, 187-197.

Koch BC, Nagtegaal JE, Hagen EC, Wee PM \& Kerkhof GA (2010): Different melatonin rhythms and sleep-wake rhythms in patients on peritoneal dialysis, daytime hemodialysis and nocturnal hemodialysis. Sleep Med 11, 242-246.

Lenz ER, Pugh LC, Milligan RA, Gift A \& Suppe F (1997): The middle-range theory of unpleasant symptoms: an update. ANS Adv Nurs Sci 19, 14-27.

Lieberman JA (2007): Update on the safety considerations in the management of insomnia with hypnotics: incorporating modified-release formulations into primary care. Prim Care Companion J Clin Psychiatry 9, 25-31.

Morgan DL \& Morgan RK (2009) Single-Case Research Methods. SAGE, Los Angeles.

Morin C \& Espie C (2004) Insomnia - A clinical guide to assessment and treatment. Springer, New York.

Morin CM, Colecchi C, Stone J, Sood R \& Brink D (1999): Behavioral and pharmacological therapies for late-life insomnia: a randomized controlled trial. JAMA 281, 991-999.

Murtagh FE, Addington-Hall J \& Higginson IJ (2007): The prevalence of symptoms in endstage renal disease: a systematic review. Adv Chronic Kidney Dis 14, 82-99.

Neutel CI \& Patten SB (2009): Sleep medication use in Canadian seniors. Can J Clin Pharmacol 16, e443-452.

Noble H, Kelly D, Rawlings-Anderson K \& Meyer J (2007): A concept analysis of renal supportive care: the changing world of nephrology. J Adv Nurs 59, 644-653.

Novak M, Shapiro CM, Mendelssohn D \& Mucsi I (2006): Diagnosis and management of insomnia in dialysis patients. Semin Dial 19, 25-31.

Ossareh S, Roozbeh, J. Krishnan, M. Bargman, JM. Oreopoulos, DG (2003): Fatigue in peritoneal dialysis patients. Int Urol and Nephrol 35, 535-541.

Pinto LR, Jr., Alves RC, Caixeta E, Fontenelle JA, Bacellar A, Poyares D, Aloe F, Rizzo G, Minhoto G, Bittencourt LR, Ataide L, Jr., Assis M, Pradella-Hallinan M, Pinto MC, Rodrigues RN, Hasan R, Fonseca R \& Tavares S (2010): New guidelines for diagnosis and treatment of insomnia. Arq Neuropsiquiatr 68, 666-675.

Pisoni RL, Wikstrom B, Elder SJ, Akizawa T, Asano Y, Keen ML, Saran R, Mendelssohn DC, Young EW \& Port FK (2006): Pruritus in haemodialysis patients: International results from the Dialysis Outcomes and Practice Patterns Study (DOPPS). Nephrol Dial Transplant 21, 3495-3505.

Redwine L, Hauger RL, Gillin JC \& Irwin M (2000): Effects of sleep and sleep deprivation on interleukin-6, growth hormone, cortisol, and melatonin levels in humans. J Clin Endocrinol Metab 85, 3597-3603.

Riegel B, Carlson B, Kopp Z, LePetri B, Glaser D \& Unger A (2002): Effect of a standardized nurse case-management telephone intervention on resource use in patients with chronic heart failure. Arch Intern Med 162, 705-712. 
Riegel B \& Weaver TE (2009): Poor sleep and impaired self-care: towards a comprehensive model linking sleep, cognition, and heart failure outcomes. Eur J Cardiovasc Nurs $\mathbf{8}$, 337-344.

Sabry AA, Abo-Zenah H, Wafa E, Mahmoud K, El-Dahshan K, Hassan A, Abbas TM, Saleh Ael B \& Okasha K (2010): Sleep disorders in hemodialysis patients. Saudi J Kidney Dis Transpl 21, 300-305.

Sloan JA, Cella D \& Hays RD (2005): Clinical significance of patient-reported questionnaire data: another step toward consensus. J Clin Epidemiol 58, 1217-1219.

Stepanski EJ (2002): The effect of sleep fragmentation on daytime function. Sleep 25, 268276.

Sullivan M, Karlsson J \& Ware JE, Jr. (1995): The Swedish SF-36 Health Survey I. Evaluation of data quality, scaling assumptions, reliability and construct validity across general populations in Sweden. Soc Sci Med 41, 1349-1358.

Tentori F, Elder SJ, Thumma J, Pisoni RL, Bommer J, Fissell RB, Fukuhara S, Jadoul M, Keen ML, Saran R, Ramirez SP \& Robinson BM (2010): Physical exercise among participants in the Dialysis Outcomes and Practice Patterns Study (DOPPS): correlates and associated outcomes. Nephrol Dial Transplant 25, 3050-3062.

Walters AS, LeBrocq C, Dhar A, Hening W, Rosen R, Allen RP \& Trenkwalder C (2003): Validation of the International Restless Legs Syndrome Study Group rating scale for restless legs syndrome. Sleep Med 4, 121-132.

Ware JE, Jr. \& Sherbourne CD (1992): The MOS 36-item short-form health survey (SF-36). I. Conceptual framework and item selection. Med Care 30, 473-483.

WHO (1993) The ICD-10 Classification of Mental and Behavioural Disorders: Diagnostic Criteria for Research WHO, Geneva.

WMA (2008) World medical association declaration of helsinki. Etical principles for medical research involving human subjects. Available at: http://www.wma.net/en/30publications/10policies/b3/index.html.

Wolfe F \& Michaud K (2010): The Hawthorne effect, sponsored trials, and the overestimation of treatment effectiveness. J Rheumatol 37, 2216-2220.

Wong FK, Chow SK \& Chan TM (2010): Evaluation of a nurse-led disease management programme for chronic kidney disease: a randomized controlled trial. Int J Nurs Stud 47, 268-278.

Yang JY, Huang JW, Peng YS, Chiang SS, Yang CS, Yang CC, Chen HW, Wu MS, Wu KD, Tsai TJ \& Chen WY (2007): Quality of sleep and psychosocial factors for patients undergoing peritoneal dialysis. Perit Dial Int 27, 675-680.

Yellen SB, Cella DF, Webster K, Blendowski C \& Kaplan E (1997): Measuring fatigue and other anemia-related symptoms with the Functional Assessment of Cancer Therapy (FACT) measurement system. J Pain Symptom Manage 13, 63-74.

Yngman-Uhlin P \& Edell-Gustafsson U (2006): Self-reported subjective sleep quality and fatigue in patients with peritoneal dialysis treatment at home. Int J Nurs Pract 12, 143152. 


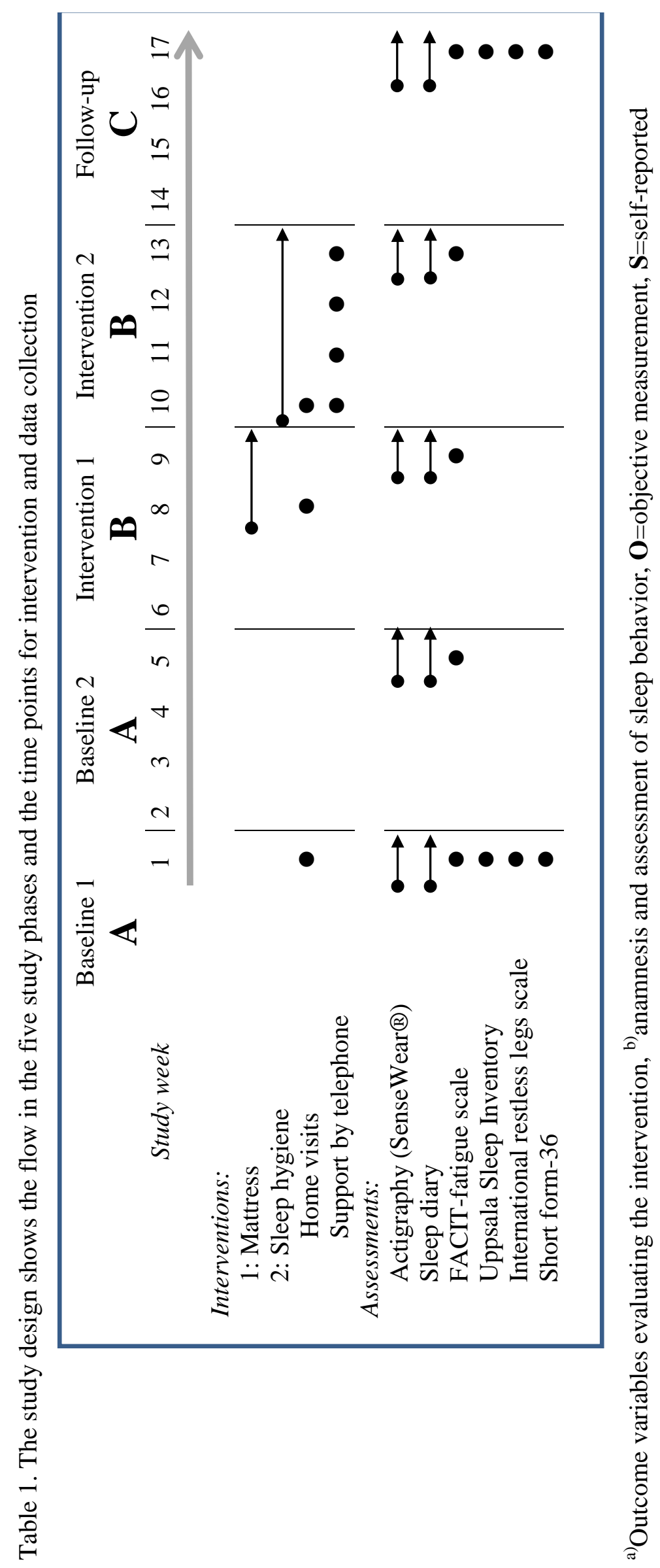




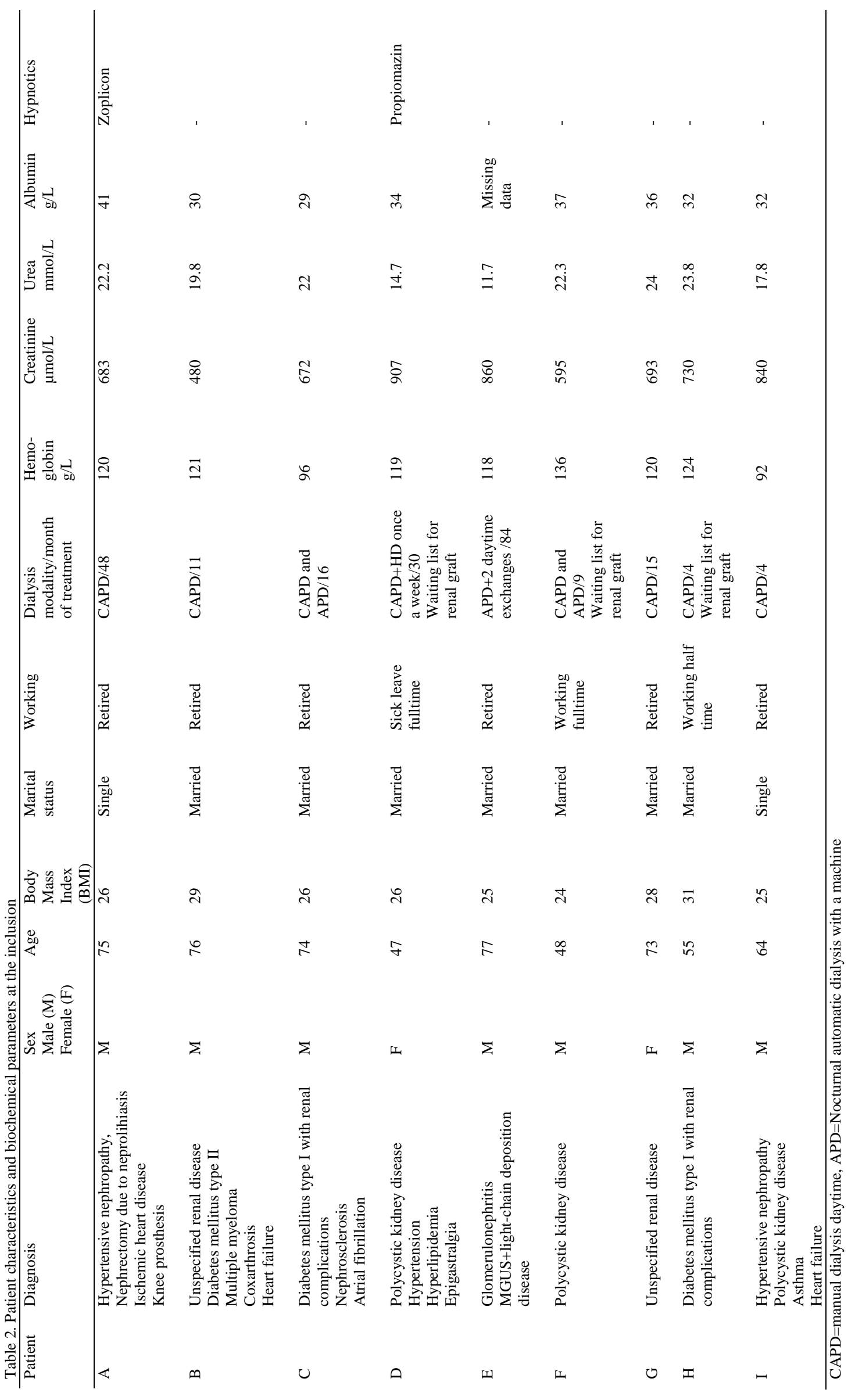




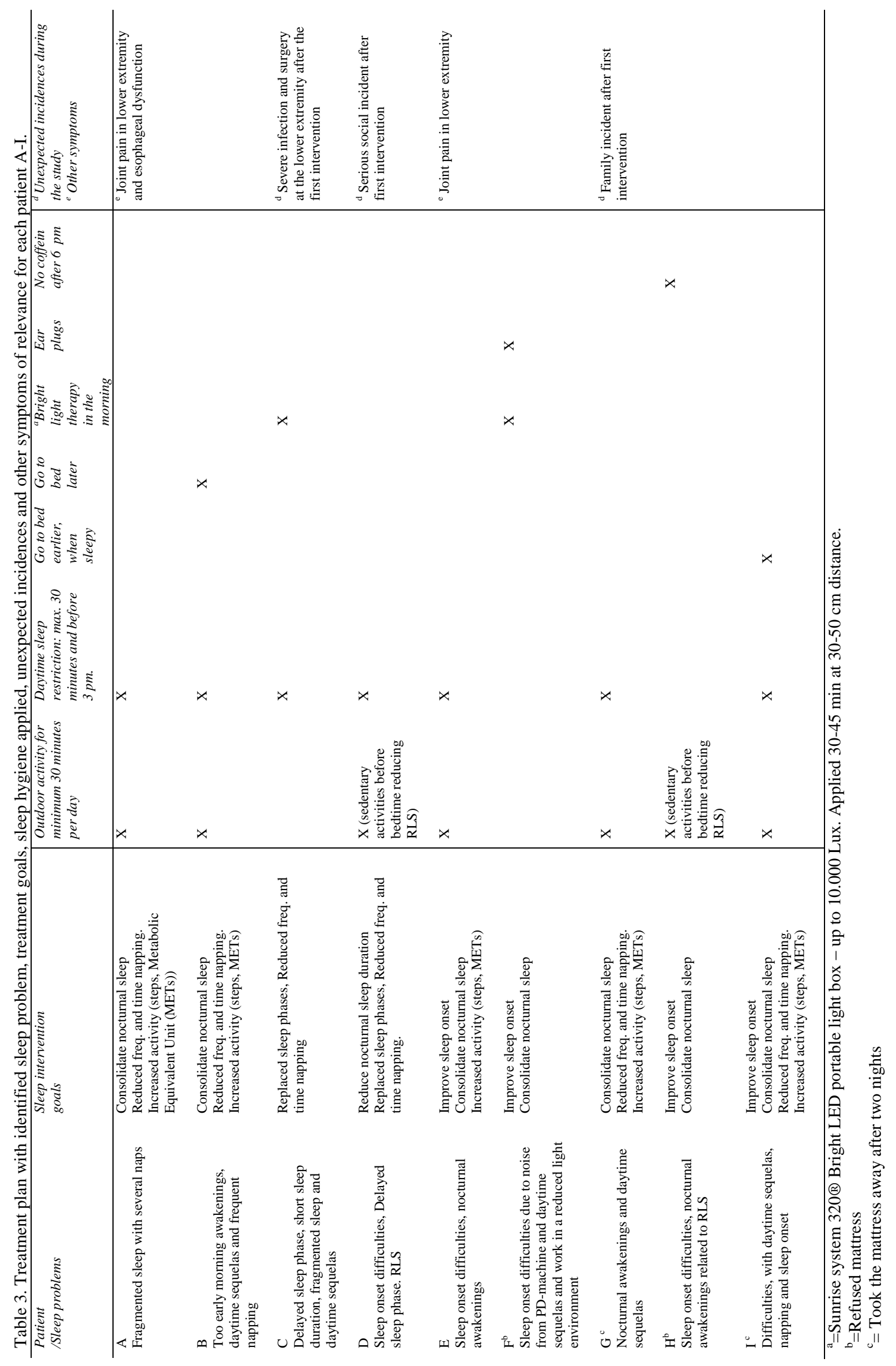




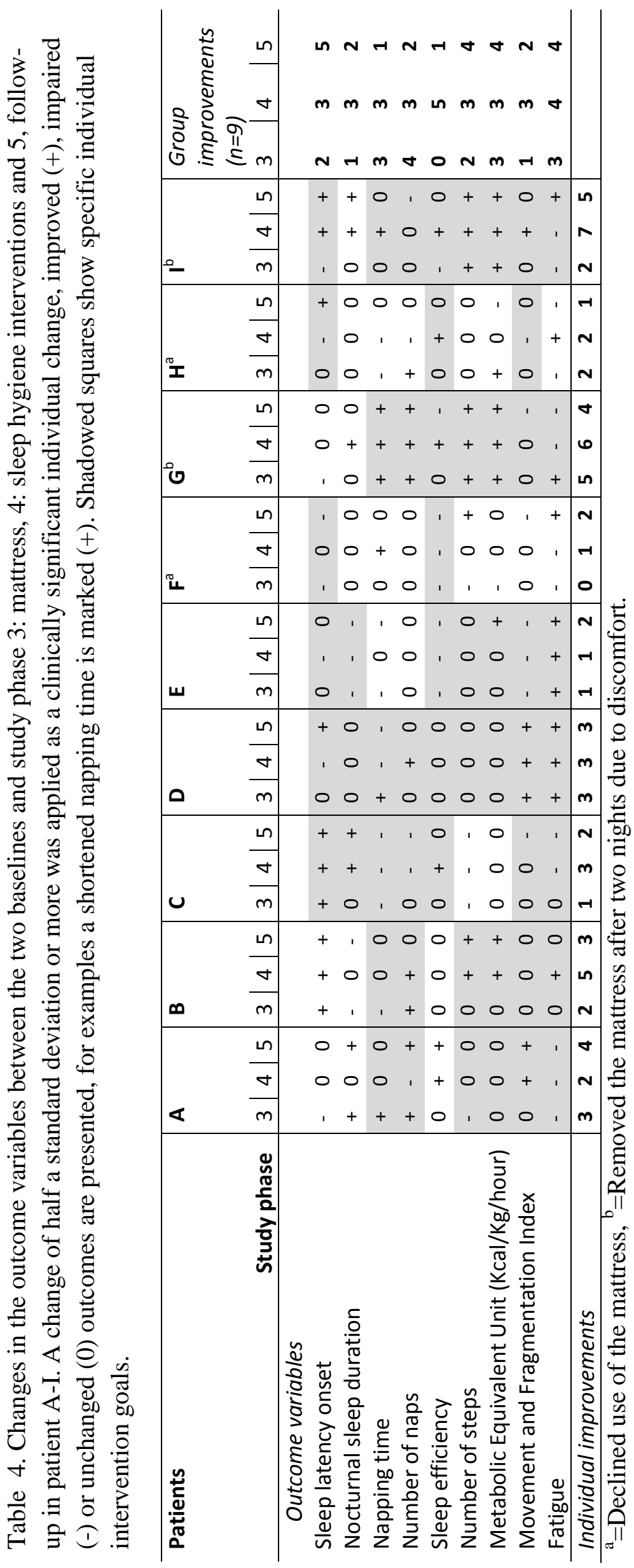



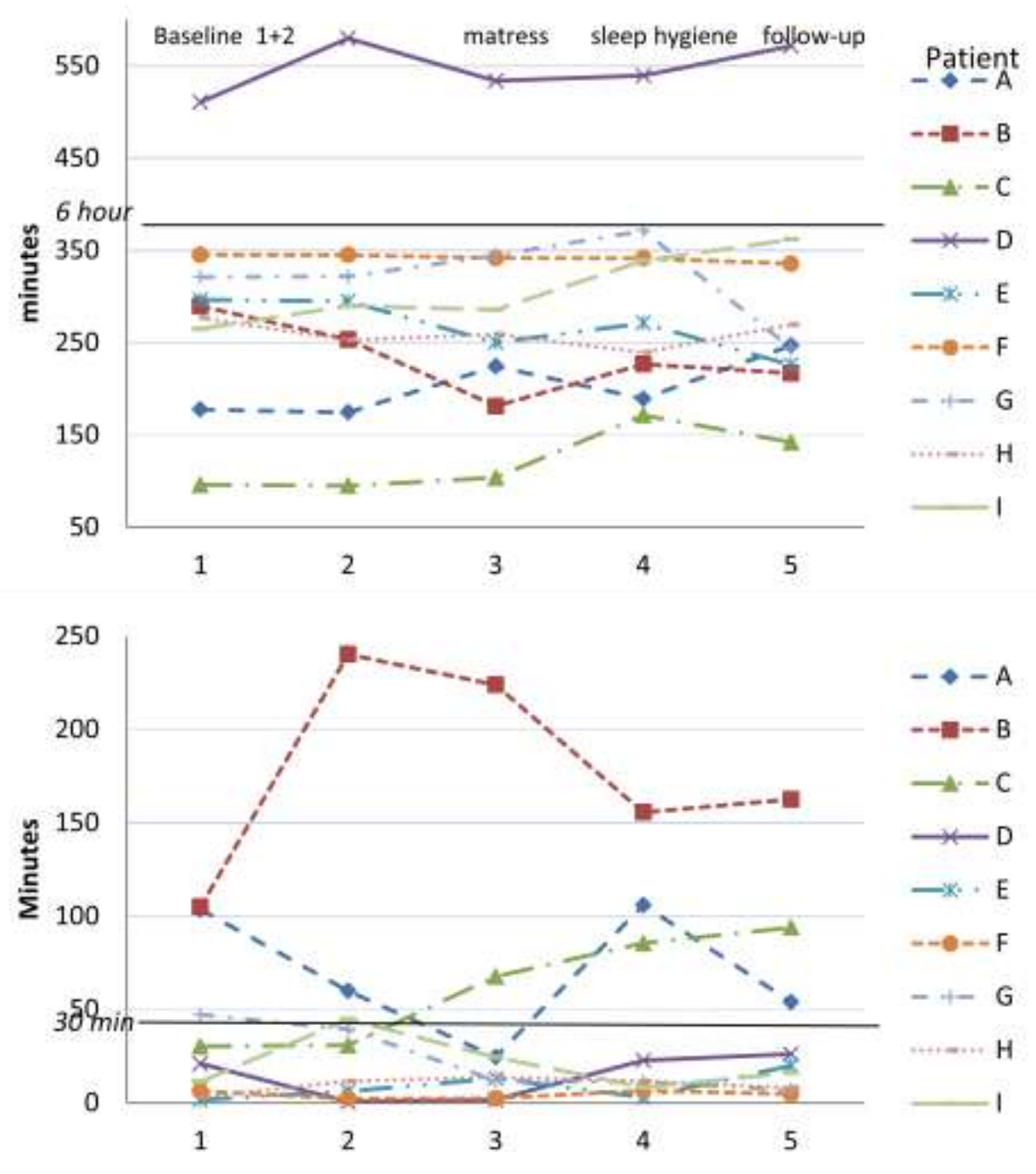

Figure 1. Illustrations of nocturnal sleep duration (top) and napping time (bottom) for patient A-I during the study phase 1-5. Nocturnal sleep duration 6 hours or more and daytime napping less than 30 minutes is recommended if Insomnia is persisting. Note that this is not a continuous assessment, the lines between the dots is just to visualize the changes for each patient. 


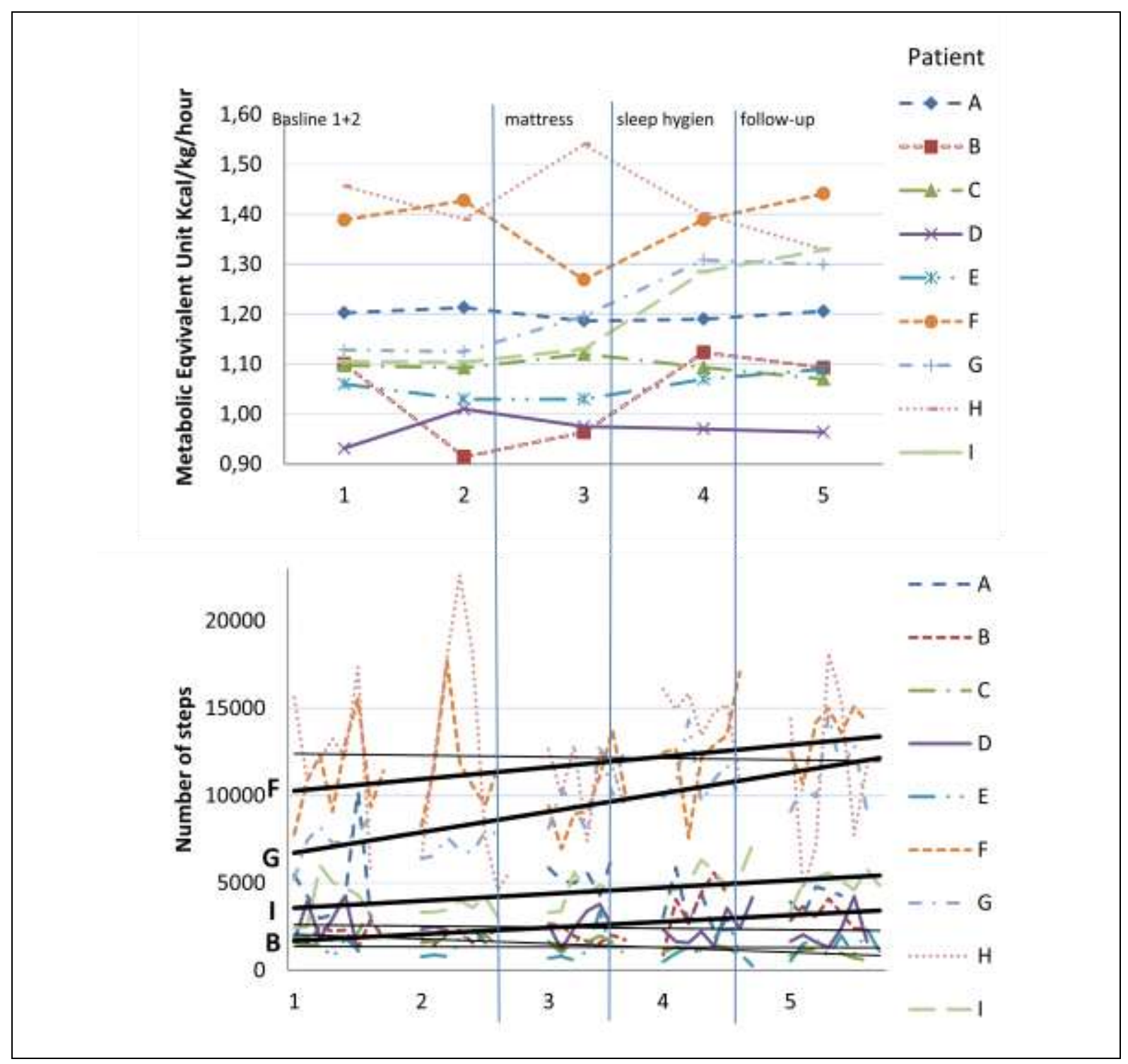

Figure 2. Illustrations of changes in Metabolic Equivalent Unit (METs)(top) and number of steps (bottom) for patient A-I, during the five study phases. Four patients had clinical significantly improvements, marked out with bold lines. Note that this is not a continuous assessment, the lines between the dots is just to visualize the changes for each patient. 


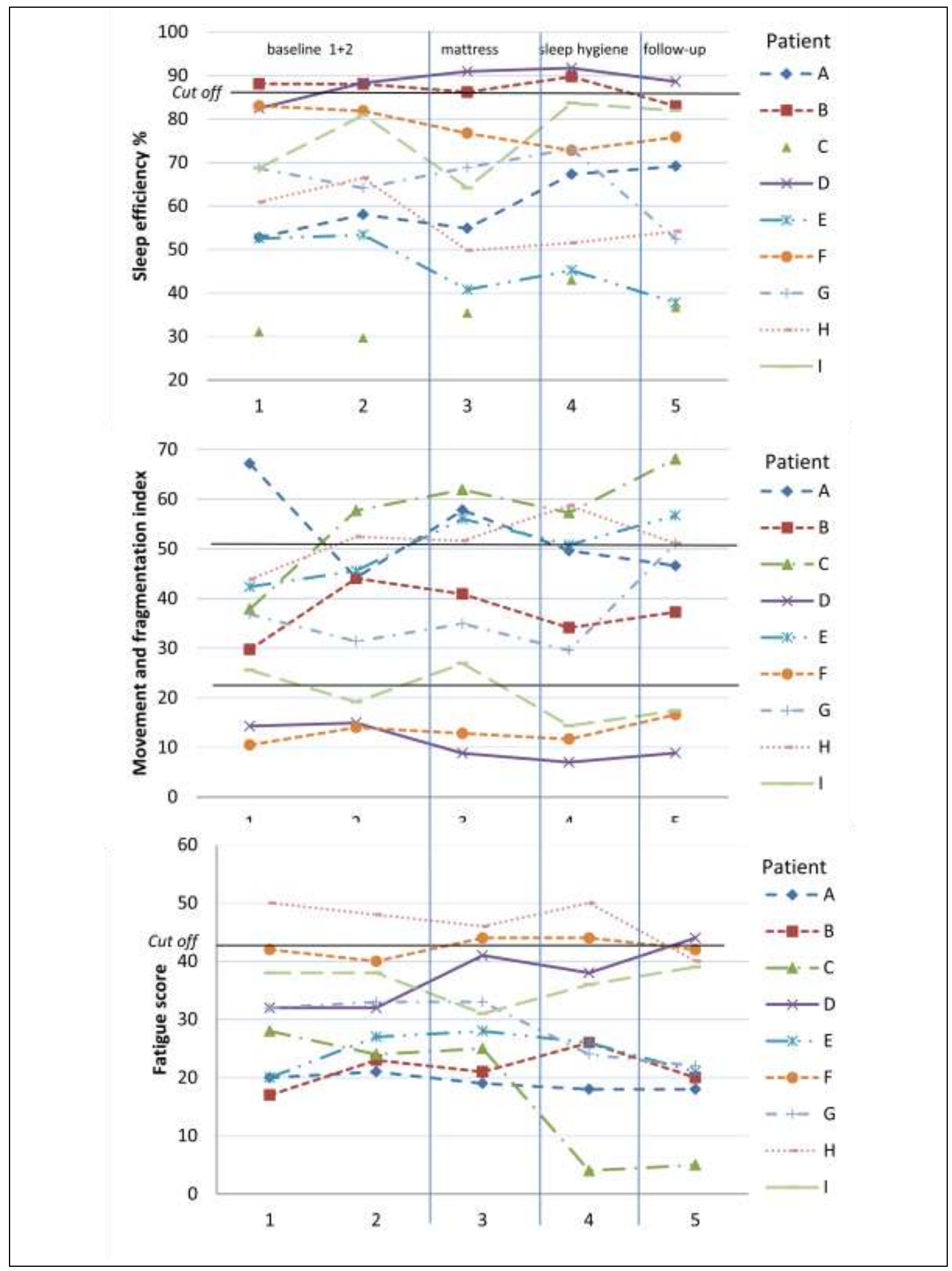

Figure 3. Illustrations of changes in sleep efficiency (above), movement and fragmentation index (MFI) (middle) and fatigue score (below) in patient A-I during the five study phases. Sleep efficiency more than $85 \%$ and MFI below 20 are well consolidated sleep. MFI above 50 are indicating seriously 

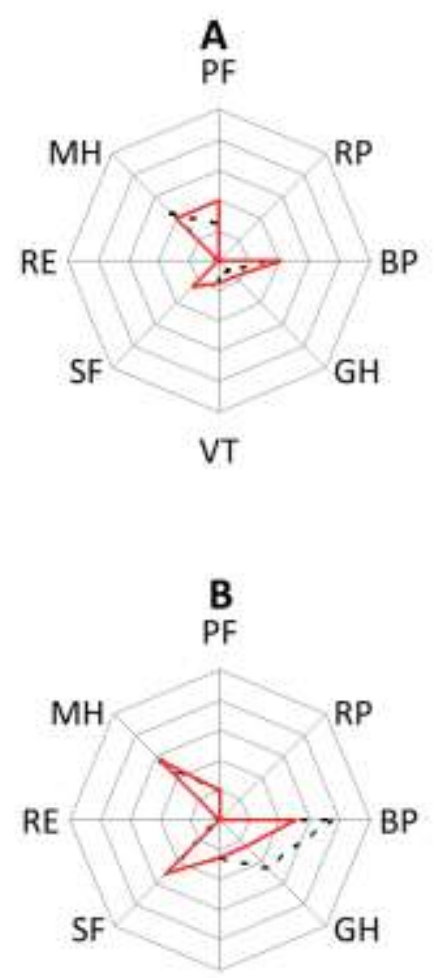

VT

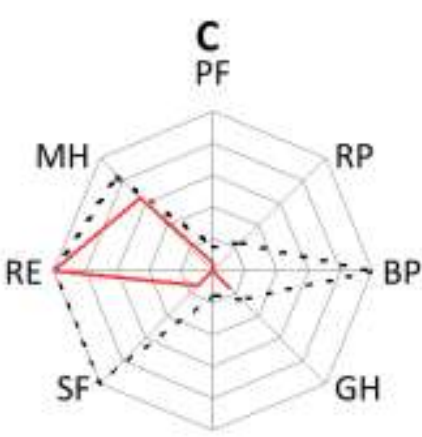

VT

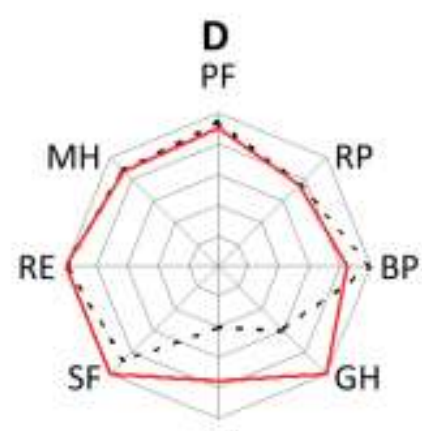

VT

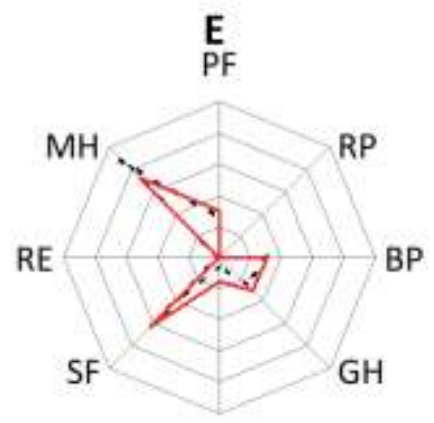

VT

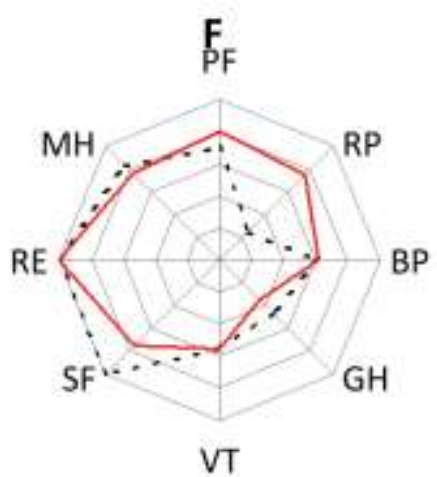

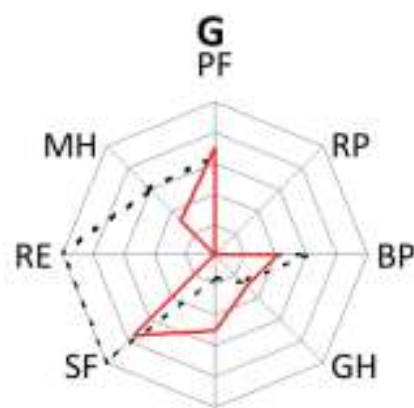

VT

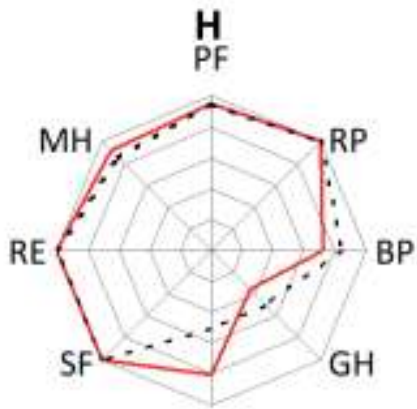

VT

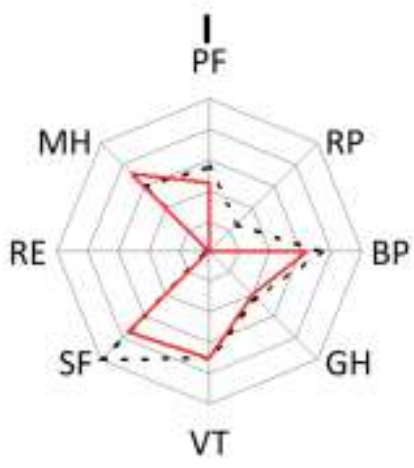

Figure 4. Health profiles (SF-36) from baseline 1 (dotted line) to follow-up (continuous line) in patient A-I. A narrow area indicates poor health and a wide area indicates good health. $\mathrm{PF}=$ physical functioning, $\mathrm{RP}=$ role limitations due to physical health problems, $\mathrm{BP}=$ bodily pain, $\mathrm{GH}=$ general health, $\mathrm{VT}=$ vitality, $\mathrm{SF}=$ =social function, $\mathrm{RE}=$ =role limitations due to emotional problems and $\mathrm{MH}=$ mental health. Scale: 0 (centre)-100. 